\title{
Simultaneous PET-MRI studies of the concordance of atrophy and hypometabolism in syndromic variants of Alzheimer's disease and frontotemporal dementia: an extended case series
}

*Kuven K Moodley ${ }^{1}$, *Daniela Perani ${ }^{2}$, Ludovico Minati ${ }^{1,3}$, Pasquale Anthony Della Rosa ${ }^{4}$, Frank Pennycook $^{5}$, John C Dickson ${ }^{6}$, Anna Barnes ${ }^{6}$, Valeria Elisa Contarino ${ }^{7}$, Sofia Michopoulou ${ }^{7}$, Ludovico D'Incerti ${ }^{7}$, Catriona Good ${ }^{8}$, Federico Fallanca ${ }^{2}$, Emilia Giovanna Vanoli ${ }^{2}$, Peter $\mathrm{J} \mathrm{Ell}^{6}$, and Dennis Chan ${ }^{1}$

*These authors equally contributed to the work

${ }^{1}$ Brighton and Sussex Medical School, Falmer, UK

${ }^{2}$ Vita-Salute San Raffaele University, Nuclear Medicine Unit San Raffaele Hospital, Division of Neuroscience IRCCS San Raffaele, Milano, Italy

${ }^{3}$ Scientific Department, Fondazione IRCCS Istituto Neurologico Carlo Besta, Milano, Italy ${ }^{4}$ Institute of Molecular Bio-imaging and Physiology, National Research Council, Milano, Italy ${ }^{5}$ Open University, Milton Keynes, UK

${ }^{6}$ Institute of Nuclear Medicine, University College London, London, UK

${ }^{7}$ Neuroradiology Department, Fondazione IRCCS Istituto Neurologico Carlo Besta, Milano, Italy

${ }^{8}$ Hurstwood Park Neurosciences Centre, West Sussex, UK

Corresponding Author:

Dr Dennis Chan

Herchel Smith Building for Brain and Mind Sciences

Department of Clinical Neurosciences

University of Cambridge

Forvie Site, Robinson Way

Cambridge CB2 0SZ

Email: dc598@medschl.cam.ac.uk

$\begin{array}{lll}\text { Word count } & \text { Abstract } & 278 \\ & \text { Main Text } & 4969\end{array}$

Tables 1

Figures 6

Supplementary Figures 6 


\begin{abstract}
Aims. The primary objective was to determine the concordance of brain atrophy and hypometabolism in six syndromic variants of Alzheimer's disease (AD) and frontotemporal dementia spectrum (FTD). A second objective was to determine the effect of image analysis methods on identification of atrophy and hypometabolism by comparing the changes observed through qualitative rating with those detected by quantitative methods.

Method. PET and MRI data were acquired simultaneously on 24 subjects with six variants of AD and FTD ( $\mathrm{n}=4$ per group). Atrophy on MRI was evaluated by visual rating and quantitative analyses of cortical thickness using Freesurfer. Hypometabolism on FDG-PET was rated visually and also quantified using the atlas-based NEUROSTAT method and a novel SPM-based approach that was recently validated. Concordance was measured using weighted Cohen's kappa.

Results. Concordance of atrophy and hypometabolism differed markedly between patient groups, with kappa scores ranging from 0.13 (for nonfluent/agrammatic variant of primary progressive aphasia, nfvPPA) to 0.49 (for the posterior cortical variant of AD, PCA). Heterogeneity was also observed within groups, with the confidence intervals of the kappa scores ranging from $0-0.25$ for PCA, to 0.29-0.61 for nfvPPA. More widespread changes on MRI and PET were identified using quantitative methods, which were also associated with superior inter-rater reliability. For quantitative PET, SPM-based evaluation identified hypometabolism in an extra $81 \%$ of brain regions when compared to the NEUROSTAT method.

Conclusion. The marked differences in concordance between syndromic variants of AD and FTD may reflect differences in underlying molecular pathologies but also operational differences in the methods used to diagnose these syndromes. The superior ability of quantitative methodologies to detect MRI and PET changes may favour their usage over current qualitative visual inspection in future clinical diagnostic practice.
\end{abstract}

\title{
Keywords
}

Simultaneous PET-MRI; Atrophy; Hypometabolism; Alzheimer's disease (AD); Frontotemporal dementia (FTD); Statistical Parametric Mapping (SPM); NEUROSTAT; Visual rating 


\section{Introduction}

In neurodegenerative dementias, MRI and 18-fluorodeoxyglucose PET (18-FDG PET) represent the respective tools of choice for detecting brain atrophy and hypometabolism. These imaging changes serve as surrogate markers of neurodegenerative pathology and their importance for diagnosis in vivo is reflected in the explicit reference to neuroimaging changes in updated diagnostic criteria. For instance, the new criteria for Alzheimer's disease (AD) [1] differ from the previous criteria [2] in specifying the presence of hippocampal atrophy on MRI and/or temporoparietal hypometabolism on FDG-PET. In the case of behavioural variant FTD (bvFTD), imaging changes are central to determination of diagnostic probability, with imaging evidence of frontal lobe damage, in the form of atrophy and/or hypometabolism, required to increase diagnostic confidence from "possible" to "probable" [3]. Similarly, the new diagnostic criteria for the variants of FTD presenting with different types of aphasia include neuroimaging changes as supportive features [4]. However a noteworthy difference between the AD and FTD diagnostic criteria is that for the former the neuroimaging changes are included as markers of AD pathophysiology whereas for the latter they are not specific to the underlying molecular pathologies.

In contrast to the abundance of single-modality studies using MRI or PET [5], relatively few multimodal studies have sought to compare patterns of brain atrophy and hypometabolism in different dementias. Those studies published to date have shown marked inter-regional differences in atrophy and hypometabolism, as exemplified by the observation that in $\mathrm{AD}$ hypometabolism is more widespread than atrophy $[6,7,8,9]$. In FTD the overlap of MRI and PET changes differ between syndromic variants, with the divergent patterns of atrophy and hypometabolism in bvFTD [10,11] contrasting with the high degree of overlap associated with the semantic variant of primary progressive aphasia (formerly known as semantic dementia) [12,13].

To date, multimodal imaging studies have tended to involve either analysis of individual disorders, or comparison across two dementia syndromes. In this extended case series simultaneous acquisition of PET and MRI on an integrated scanner is used to extend the scope of investigation to encompass six dementia syndromes, representing variants of AD and FTD. These disorders are particularly well suited for this study not only because abnormalities on MRI or PET are central to diagnosis, but also in view of the contrast between AD and FTD in terms of the relationship between molecular pathology and clinical phenotype. While in AD the same amyloid/tau proteinopathy can manifest as distinct clinical variants [14,15], in FTD a given syndromic variant may be associated with diverse underlying molecular pathologies (for example, bvFTD which can occur in the context of tau, TDP-43 or FUS protein deposition [16]).

The primary study hypothesis was that different syndromic variants of AD and FTD would be 
associated with different concordance of atrophy and hypometabolism. Above and beyond the evidence that hypometabolism both precedes atrophy in these disorders, as shown in at risk individuals [19], and exceeds the latter in terms of topographical extent $[6,7,8,9,10,11]$, it is hypothesised that these differing relationship between molecular pathology and phenotype would be reflected in between- and within-syndrome differences in concordance and this was tested across six syndromic variants (two AD and four FTD syndromes).

The second study hypothesis was that determination of the extent of atrophy and hypometabolism would differ according to the use of qualitative or quantitative evaluation methods. Proof of hypothesis would have significant implications for clinical practice given that this typically involves qualitative visual inspection of scans, in contrast to the use of quantitative methodologies in research. This study therefore compared the extent of cerebral atrophy and hypometabolism as determined by qualitative visual analysis with that identified using quantitative methodologies at a single subject level. For MRI, FreeSurfer was used to measure cortical thickness, whereas quantitative PET analyses were undertaken both with the established atlas-based NEUROSTAT method and with a recently validated voxel-based approach for single subject FDG PET analysis based on optimized statistical parametric mapping [20, 21].

\section{Methods}

\subsection{Subjects}

Ethics approval for this study was obtained from the UK South East Coast Brighton and Sussex National Research Ethics Service (reference number: 12/LO/0356). Twenty four patients with mildto-moderate dementia (clinical dementia rating of 1-2) [21] were recruited from the Cognitive Disorders Clinic, Hurstwood Park Neurosciences Centre, Haywards Heath, UK. Ten age-matched cognitively normal control subjects were recruited for comparative purposes. All patients underwent routine laboratory investigations and independent clinical assessments by two physicians expert in dementia diagnosis (DC, KKM).

Subjects were divided into six groups ( $\mathrm{n}=4$ per group). These were: typical Alzheimer's disease(AD), atypical $\mathrm{AD}$ associated with posterior cortical atrophy (PCA), behavioural variant FTD (bvFTD), nonfluent/agrammatic variant of primary progressive aphasia (nfvPPA), semantic variant of primary progressive aphasia (svPPA) and right temporal variant of FTD (rtvFTD). For all except rtvFTD diagnoses were made according to diagnostic guidelines [1,3,4]; at present there are no guidelines or criteria for the diagnosis of rtvFTD and patients with this disorder were diagnosed on the basis of focal, predominantly right-sided, temporal lobe atrophy in line with previously published studies $[22,23]$.

The rtvFTD patients in this study presented with early, prominent neuropsychiatric symptoms and 
impairments in social cognition, memory, spatial orientation and prosopagnosia resulting in altered social and occupational functioning; the diagnosis of bvFTD in these individuals was precluded by the presence of early spatiotemporal disorientation and memory impairment, [3].

Demographic and clinical data are reported in Table 1; full psychometric data are provided in Supplementary Table 1 for 20 of the 24 subjects with dementia for whom they were available. To avoid issues of circularity, it is important to clarify that while imaging data were acquired for clinical diagnostic purposes, diagnoses were made on the basis of changes observed either on MRI or on PET, in other words the concordance of MRI and PET changes was neither investigated nor considered for diagnosis.

Table 1: Demographic data

\begin{tabular}{|l|l|l|l|l|l|l|l|l|}
\hline & HC & AD & PCA & bvFTD & nfPPA & svPPA & rtvFTD & p-value \\
\hline $\mathrm{n}$ & 10 & 4 & 4 & 4 & 4 & 4 & 4 & \\
\hline $\begin{array}{l}\text { Age, mean } \\
\text { (SD) }\end{array}$ & $68.0(3.2)$ & $75.0(8.8)$ & $63.8(2.5)$ & $64.8(7.9)$ & $73.3(12.7)$ & $69.5(4.0)$ & $65.8(6.9)$ & 0.37 \\
\hline $\begin{array}{l}\text { Sex, M:F } \\
\text { Years of } \\
\text { education }\end{array}$ & $4: 6$ & $4: 0$ & $3: 1$ & $2: 2$ & $3: 1$ & $3: 1$ & $2: 2$ & 0.58 \\
\hline $\begin{array}{l}\text { CDR (2:1) } \\
\text { N/A }\end{array}$ & $2: 2.5)$ & $12.3(2.1)$ & $12(2.9)$ & $11.25(2.5)$ & $12.5(3.2)$ & $13.5(1.7)$ & $11.8(2.4)$ & 0.72 \\
& & & $2: 2$ & & & & & \\
\hline MMSE & & $24.5(2.4)$ & $23 . .8(4.5)$ & $26.5(1.7)$ & N/A & N/A & $28(1.4)$ & 0.18 \\
\hline
\end{tabular}

KEY: $\mathrm{M}=$ Male, $\mathrm{F}=$ Female, $\mathrm{CDR}=$ Clinical dementia rating scale, MMSE=Mini mental state examination.

$\mathrm{CDR}(2: 1)$ refers to the proportion of patients with $\mathrm{CDR}=2$ and $\mathrm{CDR}=1$ respectively in each of the dementia sub-groups

NB. MMSE was not routinely assessed in all patients with nfvPPA and svPPA.

\subsection{Simultaneous PET/MRI acquisition}

PET and MRI data were acquired simultaneously on an integrated Siemens Biograph mMR PET/MR scanner, consisting of a 3T MRI scanner with integrated PET detector assembly, University College London Hospital, UK. MRI was performed, acquiring both volumetric T1-weighted (1x1x1 mm voxel size, 240x256 mm FoV, TI = $900 \mathrm{~ms}, \mathrm{TE}=2.98 \mathrm{~ms}$ ) and T2-weighted sequences (slice thickness $4 \mathrm{~mm}, 256 \times 256 \mathrm{~mm}$ FoV, TR $=4780 \mathrm{~ms}$, TE $=101 \mathrm{~ms}$ ). MR-based attenuation correction was derived from the 2-point DIXON gradient echo sequence [24].

$18^{\mathrm{F}}$-FDG PET imaging was obtained in dementia subjects only due to ethical constraints. PET images 
(1.4 x 1.4 voxel size, $2.03 \mathrm{~mm}$ slice thickness) were reconstructed using Ordered Subset Expectation Maximization (OSEM), 21 subsets, 3 iterations 5mm FWHM Gaussian smoothing, matrix 256x256, zoom $=2[25]$.

\subsection{MRI Analysis}

Qualitative analysis

Two neuroradiologists (LDI, CG) with experience in dementia imaging were blinded to clinical diagnosis. Visual ratings of brain atrophy were independently generated for the following regions of interest (ROIs): frontal lobe, hippocampus, temporal pole, perisylvian region and parietal lobe. This choice of ROIs was specifically made to reflect the raters' standard clinical diagnostic practice and the fact that visual analogue rating scales already exist for several ROIs (hippocampus, frontal lobe, parietal lobe) $[26,27,28]$. Atrophy ratings, averaged across the two raters, were scored using a fourpoint scale (0-3, where 3 indicates severe atrophy).Here, the focus was on volume loss and white matter hyperintensities were not considered.

\section{Cortical thickness quantification}

Cortical thickness analysis was performed using the FreeSurfer workflow (Massachusetts General Hospital, Harvard University, Boston MA, USA), which involves reconstruction of the white-gray matter interface and pial surface, followed by labelling based on non-linear morphing to a probabilistic brain atlas. The Freesurfer workflow is detailed elsewhere [29]. Visual verification and manual clean-up of the segmentations was performed by a specialized operator unaware of disease status. Starting from Desikan atlas [30] measurements, the following ROIs were considered: frontal lobe, lateral temporal lobe, temporal pole (TP), medial parietal lobe / precuneus ( $\mathrm{Pc}$ ), lateral parietal lobe (LP), occipital lobe, anterior cingulate gyrus and posterior cingulate gyrus. For ROIs comprising more than one region (frontal lobe, lateral temporal lobe, lateral parietal lobe and occipital lobe) cortical thickness measurements were averaged. For comparison with visual ratings, Z-scores for comparison between each patient and controls were remapped on a 0-3 discrete scale $(0=\mathrm{z}<2 ; 1=$ $2<z<3 ; 2=3<z<4 ; 3=z>4)$.

\section{Hippocampal volumetry}

Hippocampal segmentation was performed using the FSL/FIRST tool (FMRIB, Oxford Centre for Functional Magnetic Resonance Imaging of the Brain, Oxford, UK) [31], and all segmentations were visually verified. One bvFTD patient (case 13) was excluded due to poor image quality. FSL 
hippocampal segmentation was chosen over FreeSurfer because preliminary expert evaluation revealed that the latter tended to include parts of other medial temporal structures such as the parahippocampal gyrus and amygdala, and as such was not reliable on our data. The accuracy of FreeSurfer vs. FSL hippocampal segmentation is matter of ongoing debate [32,33,34].

\subsection{FDG-PET Analysis}

\section{Qualitative analysis}

Three nuclear medicine consultants experienced in dementia imaging (DP, EGV, FF) were blinded to clinical diagnosis. Visual ratings of FDG-PET uptake maps (as produced by the scanner, without additional normalization) as presence of hypometabolism were independently generated for the following ROIs: frontal lobe, lateral temporal lobe, temporal pole (TP), hippocampus (Hc)/ medial temporal lobe (MTL), medial parietal lobe/ precuneus (Pc), lateral parietal lobe (LP), occipital lobe, anterior cingulate gyrus and posterior cingulate gyrus. This set of ROIs was chosen to reflect the raters' standard clinical diagnostic practice. As above, visual ratings were scored using a four-point severity scale (0-3 where 3 represents severe hypometabolism), which was averaged across raters.

\section{NEUROSTAT}

Parametric analysis of FDG-uptake yielding z-scores for the comparison between each patient and a controls template was undertaken using the Neurological Statistical Image Analysis package (NEUROSTAT; University of Seattle, WA, USA), as detailed in [35]. In brief, scans were transformed and realigned to the Talairach and Tournoux atlas [36] and then surface rendered using 3D stereotactic surface projections to demonstrate the topography of hypometabolism. Surface rendered reads were normalized using a pons reference region, and compared to an age-stratified, normative reference database.

\section{Statistical Parametric Mapping}

Parametric analysis of FDG-uptake was also performed using voxel-level statistical parametric mapping(SPM5) by means of two-sample t-tests comparing each case against a control database of normalized and smoothed PET scans, comprising 112 scans (53 men and 59 women; mean age $=64.7$ years; $\mathrm{SD}=9.3$ years) from the European Alzheimer's Disease Consortium and the San Raffaele Scientific Research Institute databases (see [20] and [37] for validation procedures). In brief, each 18F-FDG PET patient scan was warped to the standard MNI space using a new 18F-FDG PET aging and dementia-specific template for spatial normalization and subsequently smoothed with an isotropic 
3D Gaussian kernel of $8 \mathrm{~mm}$ FWHM [20].Age was included as a covariate in the two sample t-test design of SPM5and global normalization of voxel values used proportional scaling to a mean voxel value of $6.5 \mathrm{mg} / 100 \mathrm{~mL} / \mathrm{min}$ [38].

The comparison between each case and the control database yielded a contrast map testing for areas with relative decreases in metabolism (i.e. hypometabolism) compared to the control population Significance values from the voxel-wise t-test were corrected for family-wise error over multiple comparisons at voxel level (threshold $\mathrm{pFWE}<0.05$ ), and a cluster extent threshold of $\geq 100$ voxels was additionally imposed.

Visual assessment of PET NEUROSTAT and SPM maps was undertaken for the following ROIs: frontal lobe, lateral temporal lobe, temporal pole (TP), hippocampus $(\mathrm{Hc}) /$ medial temporal lobe (MTL), medial parietal lobe/ precuneus (Pc), lateral parietal lobe (LP), occipital lobe, anterior cingulate gyrus and posterior cingulate gyrus. Regional reads were binarized $(0=$ no detectable hypometabolism; 1 = detectable hypometabolism reported by at least two raters); this choice reflected the manner in which parametric maps of subject-to-group comparisons are reported in clinical practice.

\subsection{PET-MRI concordance}

A linear weighted Cohen's kappa statistic $[39,40]$ was used to provide an adaptable metric of the concordance of atrophy and hypometabolism given the requirement to compare diverse PET and MRI datasets. A 4x2 normalised weight matrix was applied in order to relate binarised (0-1) and scaled (03) scores consistently. Kappa is used in situations when an association is expected to exist in order to quantify the degree of agreement or concordance beyond that expected by chance. In these disorders atrophy and hypometabolism are known to be highly correlated in general (confirmed for these study data using Fisher's exact test with resultant $\mathrm{p}<0.001$ for all patient groups) and the kappa statistic is used to provide a measure of the strength of this concordance in any particular patient or group.

\section{Results}

\subsection{Inter-rater reliability of atrophy and hypometabolism scores}

According to Cohen's kappa [39], the inter-rater reliability of qualitative atrophy ratings was 0.34 ( $\mathrm{p}<$ 0.001 ; 95\% CI: [0.24, 0.43]). According to Fleiss' kappa [41], the inter-rater reliability of qualitative hypometabolism ratings on uptake maps was 0.43 ( $p<0.001$; 95\% CI: [0.41, 0.45]). For NEUROSTAT and SPM-based analysis, this increased to 0.53 ( $p<0.001 ; 95 \%$ CI: $[0.49,0.57])$ and 0.90 ( $p<0.001 ; 95 \%$ CI: [0.86, 0.94]), respectively. 
$M R I$

Qualitative assessment revealed in all cases bilateral, and largely symmetrical, mild-moderate atrophy of the medial temporal lobe and temporal pole, with additional mild-moderate frontal atrophy and lateral parietal atrophy in cases each (representative case in Figure 1, remaining cases and score plots in Supplementary Figure 1). Cortical thickness measurements revealed additional lateral temporal atrophy in three cases, and atrophy of the precuneus and posterior cingulate gyrus in two cases.

\section{FDG-PET}

Qualitative visual FDG PET uptake reads revealed hypometabolism overall maximal in the lateral temporal and lateral parietal regions. NEUROSTAT and SPM-PET analyses both identified hypometabolism in the lateral parietal and, less markedly, in occipital regions, but additional hypometabolism in the precuneus and posterior cingulate gyrus was more consistently detected with SPM-PET.

\section{PET-MRI concordance}

The measured weighted kappa for cortical thickness and hypometabolism assessed via SPM PET was 0.17 (95\% CI: [0.00, 0.42]).Hypometabolism overall was more widely distributed across the brain than atrophy, particularly in posterior cortical regions (Supplementary Figure 1).

\subsection{Posterior Cortical Atrophy (PCA)}

$M R I$

Qualitative assessment revealed in all cases bilateral, symmetrical, moderate to severe atrophy of the lateral parietal cortex with additional, but less severe, atrophy of the frontal lobes (representative case in Figure 2, remaining cases and score plots in Supplementary Figure 2). Cortical thickness measurements identified more extensive atrophy, maximal in parietal and occipital regions, but also involving the right temporal pole and lateral temporal regions bilaterally in three cases.

\section{FDG-PET}

In all cases qualitative FDG-PET uptake reads revealed hypometabolism primarily affecting the occipital and lateral parietal regions bilaterally, with less severe hypometabolism additionally noted particularly in the lateral temporal lobe. NEUROSTAT and SPM-PET analyses similarly identified predominant parieto-occipital hypometabolism but additionally detected changes not only in left frontal but also in the precuneus and posterior cingulate regions. A comparison of these two analyses revealed that SPM-PET identified changes in nearly all ROIs where hypometabolism was observed using NEUROSTAT, but detected hypometabolismin the lateral temporal lobe and precuneus more 
consistently.

\section{PET-MRI concordance}

The measured weighted kappa for cortical thickness and hypometabolism assessed via SPM PET was 0.49 (95\% CI: [0.29, 0.61]). Overall, there was good concordance between atrophy and hypometabolism in the frontal as well as posterior cortical regions (Supplementary Figure 2), however some discordance was evident in the temporal poles and medial temporal lobe, where mild-moderate atrophy was not always accompanied by hypometabolism.

\subsection{Behavioural variant FTD}

$M R I$

Qualitative assessment revealed mild-moderate frontal lobe atrophy in all cases and, albeit less strongly and consistently, additional lateral parietal lobe and temporal lobe (temporal pole and medial temporal lobe) atrophy (representative case in Figure 3, remaining cases and score plots in Supplementary Figure 3). Compared to the qualitative reads, cortical thickness measurements revealed marked inter-individual differences in atrophy pattern; in two cases there was highly restricted or no atrophy measured, whereas in the remaining two cases atrophy was observed in the frontal regions with involvement of medial temporal or parietal regions.

\section{FDG-PET}

Qualitative PET uptake readings revealed hypometabolism primarily in the frontal lobes but with differing degrees of severity; no frontal hypometabolism was observed in one case (Case 12) whereas in another case (Case 11) moderate to severe hypometabolism was observed not only in the frontal lobes but also in lateral parietal regions. NEUROSTAT and SPM-PET analyses overall revealed significant changes in the frontal lobes and anterior cingulate gyri in all cases; in particular highlighting anterior cingulate involvement not detected visually in one case (Case 12) and diffuse parietal involvement in another (Case 11).

\section{PET-MRI concordance}

The measured weighted kappa for cortical thickness and hypometabolism assessed via SPM PET was 0.22 (95\% CI: $[-0.11,0.47])$. For this subgroup, the overlap appeared highly variable across individuals, as exemplified by the high co-localization of hypometabolism and cortical thinning in Case 11 and the observation of significant hypometabolism in multiple regions without corresponding atrophy in Case 12, (Supplementary Figure 3).

\subsection{Nonfluent/agrammatic variant of primary progressive aphasia}


Qualitative assessment revealed in all cases bilateral, mild-moderate atrophy of the frontal and parietal regions, with additional atrophy of the medial temporal pole observed in two cases (representative case in Figure 4, remaining cases and score plots in Supplementary Figure 4). Cortical thickness measurements revealed marked inter-individual differences, with lateral temporal lobe (bilaterally), left precuneus and left lateral parietal atrophy jointly observed in $2 / 4$ cases and much more limited involvement of these regions in the other cases.

\section{FDG-PET}

Qualitative PET reads were characterised by overall predominantly left-sided hypometabolism that was maximal in the perisylvian regions(lateral temporal, lateral parietal and inferior frontal cortex) with additional right parietal and temporal hypometabolism less consistently observed. NEUROSTAT and SPM-PET analyses revealed a similar but more extended pattern of hypometabolism, both in the left hemisphere (e.g., Case 15) and in terms of right hemispheric involvement (e.g., Case 16).

\section{PET-MRI concordance}

The measured weighted kappa for cortical thickness and hypometabolism assessed via SPM PET was 0.13 (95\% CI: [0.00, 0.25]). Overall, hypometabolism was more extended than atrophy: notably, in the left frontal lobe and parietal regions hypometabolism was consistently observed without corresponding atrophy (Supplementary Figure 4).

\subsection{Semantic variant of primary progressive aphasia}

\section{$M R I$}

Qualitative assessment revealed in all cases moderate-severe bilateral medial temporal and temporal pole atrophy with milder degrees of atrophy observed in frontal and parietal regions (representative case in Figure 5, remaining cases and score plots in Supplementary Figure 5). Cortical thickness measurements identified more extensive atrophy, with asymmetrical, predominantly left sided temporal involvement except in one case which was relatively symmetrical (Case 20), with additional changes involving the medial parietal and the anterior and posterior cingulate regions observed in that case.

\section{FDG-PET}

Qualitative FDG-PET uptake evaluation identified diffusely left sided hypometabolism involving the medial temporal, lateral temporal and lateral parietal lobes; the distribution of hypometabolism was less asymmetrical in one case (Case 20). NEUROSTAT and SPM-PET analyses confirmed diffuse hypometabolism in the left hemisphere, particularly revealing additional precuneus and posterior 
cingulate involvement. Furthermore, SPM-PET also identified contralateral frontal, temporal and parietal changes not visible to qualitative and NEUROSTAT evaluation in three cases.

\section{PET-MRI concordance}

The measured weighted kappa for cortical thickness and hypometabolism assessed via SPM PET was 0.35 (95\% CI: [0.19, 0.44]). Overall, there was high concordance between atrophy and hypometabolism inleft temporal regions. However, discordance was evident particularly in the left lateral parietal lobe and left anterior cingulate gyrus, where hypometabolism was observed without corresponding atrophy in 3/4 cases (Supplementary Figure 5).

\subsection{Right temporal variant FTD}

\section{MRI}

Qualitative assessment revealed in all cases moderate-severe bilateral medial temporal and temporal pole atrophy with milder degrees of atrophy observed in the frontal and parietal regions bilaterally (representative case in Figure 6, remaining cases and score plots in Supplementary Figure 6). Cortical thickness measurements identified more extensive atrophy, with asymmetrical, predominantly rightsided, temporal pole and lateral temporal lobe atrophy in all cases and asymmetrical, predominantly right-sided, parietal lobe atrophy in 2 cases.

\section{FDG-PET}

Qualitative PET assessment identified right-sided temporal pole and lateral temporal hypometabolism with additional involvement of medial temporal lobe, lateral parietal and left temporal pole hypometabolism that was observed in three cases. NEUROSTAT and SPM-PET analyses identified bilateral hypometabolism of the temporal poles and lateral parietal lobes, right frontal lobe and left medial temporal region. When compared with the NEUROSTAT reads, widespread additional hypometabolism was observed with SPM-PET, encompassing the anterior cingulate gyrus bilaterally, and the right medial temporal regions in two cases.

\section{PET-MRI concordance}

The measured weighted kappa for cortical thickness and hypometabolism assessed via SPM PET was 0.35 (95\% CI: $[0.23,0.45])$. As anticipated given the focal nature of the changes, there was concordance between atrophy and hypometabolism bilaterally in the right lateral temporal lobe and temporal pole (Supplementary Figure 6). Discordance was most evident in the frontal regions and anterior cingulate gyrus where hypometabolism without atrophy was observed.

\section{Discussion}


Analysis of simultaneously acquired PET and MRI revealed marked differences in the degree of topographic overlap, or concordance, of atrophy observed on MRI and hypometabolism identified using FDG-PET in patients with syndromic variants of Alzheimer's disease (AD) and frontotemporal dementia (FTD). Concordance between cortical thickness, as measured on MRI using Freesurfer, and hypometabolism as identified using a novel SPM-based technique for quantitative PET analysis $[18,35]$ was measured using a weighted Cohen's kappa statistic, to permit the comparison of graded outputs (Freesurfer) with a binary read (SPM-PET). There was more than a threefold difference in concordance between the various AD and FTD syndromes, with kappa scores ranging from 0.13 in patients with nonfluent/agrammatic variant of primary progressive aphasia (nfvPPA) to 0.49 in patients with the posterior cortical variant of AD (PCA). The range of concordance varied also within each group of patients with different syndromes, as shown by comparing the confidence interval of the kappa scores; confidence intervals of0-0.25 for the group of patients with nfvPPA group, indicative of greater within-group heterogeneity, contrasted with scores of 0.29-0.61 for the PCA patient group.

This study at single subject level has also shown that determination of the extent of atrophy and hypometabolism in these disorders is dependent on the choice of analysis method. For MRI, quantitative measures of cortical thickness typically revealed more widespread cortical change than was detected on qualitative visual inspection, and similarly the two quantitative PET analysis methods (the atlas-based NEUROSTAT and the novel SPM-based technique) identified more widespread hypometabolism than visual inspection of FDG uptake distributions. A direct comparison of the two quantitative PET methods revealed that both methods detected hypometabolism in 108 regions of interest (ROIs), out of all the ROIs evaluated in all patients, but that the SPM-PET alone detected change in a further 88 ROIs, representing an $81 \%$ increase. These 88 ROIs were spread across all patients and all disease groups, and in all lobes of the brain, rather than being evident in selected cases or brain regions only, suggesting that the additionally detected hypometabolism is indicative of a generally higher sensitivity of the SPM-PET technique rather than due to specific pathological or anatomical considerations. This in turn may relate to the methodological differences between NEUROSTAT and SPM-PET; while the former is based on projections from an atlas, the latter involves elastic normalization to a tailored control template, followed by voxel-level inference, with attendant increase in spatial specificity[20,37].

Inter-rater reliability was also superior for quantitative PET techniques, with inter-rater reliability indices of 0.53 and 0.9 for NEUROSTAT and SPM-PET respectively comparing with a reliability index of 0.43 for visual PET reads.

Atrophy and hypometabolism reflect different aspects of pathophysiology and analysis of simultaneously acquired PET and MRI data provides an opportunity to compare and contrast patterns 
of brain involvement at different "severity stages" in the disease process. In keeping with many previous studies, this study has shown that there is divergence in the patterns of atrophy and hypometabolism, with the latter typically exceeding the former in terms of topographical extent. The occurrence in certain brain regions of hypometabolism without corresponding atrophy is consistent with the notion that in these neurodegenerative diseases, impairment of synaptic and neuronal function precedes neuronal loss and may be indicative of a stage of pathology of sufficient severity to be manifest as altered metabolism, as marker of neuronal injury, but insufficiently advanced to result in overt regional atrophy. In $\mathrm{AD}$, this has been clearly documented $[7,18,42]$ supporting also the different order in the pathological events in the AD cascade models [43]. In addition, it has been shown that a significant fraction of cortical hypometabolism is due to mechanisms other than local atrophy, such as disconnection effects [6,7]. Thus, disconnection also plays a part in the observed hypometabolism.

While this may serve to explain the differing patterns of atrophy and hypometabolism, it is insufficient to explain the between-group differences in the concordance of these imaging changes. Several alternative explanations therefore need to be considered. First of all, the heterogeneous concordances may represent differences among these syndromic variants in terms of the temporal relationship between the onset of hypometabolism and subsequently of atrophy, and in the extent of functional disconnection associated with each disorder. However, it is probable that at least part of the explanation relates to the way in which these syndromes are diagnosed clinically. Concordance was greatest in patients with PCA (kappa score 0.495) and in patients with right temporal variant FTD (rtvFTD; kappa 0.347), but in both instances the presence of imaging changes is not just diagnostically supportive but the defining characteristic for clinical diagnosis. By contrast, patients with typical $\mathrm{AD}$, in whom the kappa score was 0.167 , were diagnosed according to clinical (not research) criteria [1] which do not stipulate the requirement for imaging changes. By the same token, there was a near three-fold difference in concordance between the two variants of primary progressive aphasia (PPA), with kappa scores of 0.353 and 0.132 for semantic variant PPA (svPPA) and nonfluent/agrammatic PPA (nfvPPA) respectively. While PPA overall is diagnosed on clinical grounds [44], a more recently published classification of PPA variants [4] outlines criteria for the diagnosis of "imaging-supported" svPPA and nfvPPA. However the striking disparity in concordance of imaging changes associated with these two PPA subtypes, observable even with $n=4$ group sizes, indicates that future revisions of PPA diagnostic criteria may need to take into account the heterogeneity of functional and structural imaging changes.

Within each patient group there was marked inter-individual variability in patterns of atrophy and hypometabolism, and in their concordance, unrelated to differences in demographics, age or disease severity as determined by the Clinical Dementia Rating. The demonstration of such within-group differences is particularly relevant given the tendency to report multimodal imaging changes in AD or 
FTD variants at a group level [45]. In FTD different molecular pathologies have been found to be associated with different patterns of cerebral hypometabolism and one possible explanation for this within-group heterogeneity may therefore be that of differences in underlying molecular pathology $[46,47]$. However, in the absence of genetic or immunohistochemical confirmation such an explanation is necessarily speculative.

In this context, the finding of similar heterogeneity in $\mathrm{AD}$ (in patients considered to have the same molecular pathology) and FTD ( in patients with potentially diverse pathologies) suggest that additional factors, such as environmental, genetic or epigenetic modifiers, may influence the imaging phenotype $[16,17,18]$.

The current study has limitations that need to be acknowledged. First, there were four patients in each disease subgroup, and these small sample sizes precluded generalization through statistically-robust group-level conclusions. Instead it is proposed that this study be considered as an extended case series, the findings from which will inform the hypotheses and design of future large scale multimodal imaging studies, interest in which will be heightened by the more widespread availability of integrated PET-MRI scanners of the kind used in this study. The demonstration of clear between- and withingroup differences in spite of the small group sizes provides a compelling counter-argument to the notion that small-n studies cannot yield meaningful results. Second, any analyses of PET data in dementia studies need to consider potential confounds relating to concomitant atrophy and to the partial volume effect although with regard to the latter previous PET studies in AD have demonstrated that the observation of reduced FDG uptake in crucial brain regions remained robust independent of any partial volume correction [48]. Comparison of MRI and PET data also needed to take into account the different approaches to scoring change. In accordance with clinical practice qualitative assessments of MRI and PET employed a severity scale ranging from 0 (absent) to 3 (severe); in contrast NEUROSTAT- and SPM-PET outputs are in the form of binary reads (absent or present). For this reason concordance of atrophy and hypometabolism was determined using a weighted Cohen's kappa, which unlike other measures of similarity such as the Jaccard index, can be scaled to deal with comparison between binary and many-valued scores.

Finally, the control groups were different for SPM-PET, NEUROSTAT and FreeSurfer. This is a reflection of the different image analysis methodologies; NEUROSTAT has a standard set of controls which is nearly always utilized with the tool, whereas for SPM-PET patient data were compared against an extensive, locally acquired, template and controls dataset [20,21]. However an equivalent dataset was not available for MRI and so MRI control data were obtained from a separate control group who were scanned on the study scanner as the study patients.

\section{Conclusion}

Two main findings arose from this study. The concordance of atrophy and hypometabolism differed 
markedly across syndromic variants of AD and FTD. This heterogeneity is considered to reflect not only differing underlying molecular pathologies but also operational differences in the criteria used to diagnose these syndromes. In addition, the demonstration of additional heterogeneity within patient groups has implications for the current practice of describing the imaging correlates of AD and FTD at group level. We thus suggest use of metrics in single subject analysis for MRI and FDG PET as it was reported [49].

Quantitative methods identified more widespread atrophy and hypometabolism than qualitative visual ratings and for the PET analyses in single individuals, the most extensive change was detected using a novel SPM-based technique [20,21]. These observations, in combination with their superior inter-rater reliability, have implications for the future implementation of quantitative methodologies in clinical practice, and through use of integrated PET/MRI scanners.

\section{Acknowledgements}

The authors would like to thank the patients and control subjects for their participation, and the Trustees of University College London, who funded the PET-MRI scans. Thanks also to Celia O'Meara and Laura Bottomley, for their help with study administration, and to Dr Stuart Anderson for his input into the interpretation of neuropsychological results.

The SPM-PET analyses for the study were funded by EU FP7 INMIND Project (FP7-HEALTH-201, grant agreement no. 278850) to DP.

\section{References}

1. McKhann GM, Knopman DS, Chertkow H, Hyman BT, Jack CR, Kawas CH, et al. The diagnosis of dementia due to Alzheimer's disease: Recommendations from the National Institute on Aging-Alzheimer's Association workgroups on diagnostic guidelines for Alzheimer's disease. Alzheimers Dement 2011; 7:263-9.

2. McKhann G, Drachman D, Folstein M, Katzman R, Price D, Stadlan EM. Clinical diagnosis of Alzheimer's disease: report of the NINCDS-ARDA Work Group under the auspices of the Department of Health and Human Services Task Force on Alzheimer's Disease. Neurology 1984; 34:939-44.

3. Rascovsky K, Hodges JR, Knopman D, Mendez MF, Kramer JH, Neuhaus J, et al. Sensitivity of revised diagnostic criteria for the behavioural variant of frontotemporal dementia. Brain 2011; 134:2456-77.

4. Gorno-Tempini ML, Hillis AE, Weintraub S, Kertesz A, Mendez M, Cappa SF, et al. Classification of primary progressive aphasia and its variants. Neurology 2011; 76:1006-14. 
5. Whitwell JLJ, Jack CRC. Neuroimaging in dementia. Neurol Clin 2007; 25:843-57.

6. Villain N, Desgranges B, Viader F, La Sayette de V, Mézenge F, Landeau B, et al. Relationships between hippocampal atrophy, white matter disruption, and gray matter hypometabolism in Alzheimer's disease. J Neurosci 2008; 28:6174-81.

7. Chételat G, Desgranges B, Landeau B, Mézenge F, Poline JB, la Sayette de V, et al. Direct voxel-based comparison between grey matter hypometabolism and atrophy in Alzheimer's disease. Brain 2008; 131:60-71.

8. La Joie R, Perrotin A, Barré L, Hommet C, Mézenge F, Ibazizene M, et al. Region-specific hierarchy between atrophy, hypometabolism, and $\beta$-amyloid $(A \beta)$ load in Alzheimer's disease dementia. J Neurosci 2012; 32: 16265-73.

9. Cerami C, Crespi C, Della Rosa PA, Dodich A, Marcone A, Magnani G, Coppi E, Falini A, Cappa SF, Perani D. Brain Changes within the Visuo-Spatial Attentional Network in Posterior Cortical Atrophy. J Alzheimers Dis. 2014 Aug 11

10. Koedam EL, Van der Flier WM, Barkhof F, Koene T, Scheltens P, Pijnenburg YA. Clinical characteristics of patients with frontotemporal dementia with and without lobar atrophy on MRI. Alzheimer Dis Assoc Disord 2010; 24: 242-7.

11.Tosun D, Rosen H, Miller BL, Weiner MW, Schuff N. MRI patterns of atrophy and hypoperfusion associations across brain regions in frontotemporal dementia. Neuroimage 2012; 59:2098-109.

12.Desgranges B, Matuszewski V, Piolino P, Chételat G, Mézenge F, Landeau B, et al. Anatomical and functional alterations in semantic dementia: A voxel-based MRI and PET study. Neurobiol Aging 2007; 28:1904-13.

13. Acosta-Cabronero J, Patterson K, Fryer TD, Hodges JR, Pengas G, Williams GB, et al. Atrophy, hypometabolism and white matter abnormalities in semantic dementia tell a coherent story. Brain 2011; 134:2025-35.

14.Galton CJ, Patterson K, Xuereb JH, Hodges JR. Atypical and typical presentations of Alzheimer's disease: a clinical, neuropsychological, neuroimaging and pathological study of 13 cases. Brain 2000; 123:484-98.

15.Lehmann M, Ghosh PM, Madison C, Laforce R Jr, Corbetta-Rastelli C, Weiner MW, Greicius MD, Seeley WW, Gorno-Tempini ML, Rosen HJ, Miller BL, Jagust WJ, Rabinovici GD.Diverging patterns of amyloid deposition and hypometabolism in clinical variants of probable Alzheimer's disease.Brain. 2013;136:844-58.

16.Josephs KA. Frontotemporal dementia and related disorders: Deciphering the enigma. Ann 
Neurol 2008; 64:4-14.

17. Whitewell JL, Josephs KA. Neuroimaging in frontotemporal lobar degeneration- predicting molecular pathology. Nat Rev Neurol 2011; 8:131-142

18. Rohrer JD. Structural brain imaging in frontotemporal dementia. Biochem Biophys Acta $2012 ; 1822: 325-332$

19.Bateman RJ, Xiong C, Benzinger TL, Fagan AM, Goate A, Fox NC, et al. Clinical and biomarker changes in dominantly inherited Alzheimer's disease. N Engl J Med 2012; 367:795-804.

20. Della Rosa PA, Cerami C, Gallivanone F, Prestia A, Caroli A, Castiglioni I, Gilardi MC, Frisoni G, Friston K, Ashburner J, Perani D; and the EADC-PET Consortium. A Standardized [18F]-FDG-PET Template for Spatial Normalization in Statistical Parametric Mapping of Dementia. Neuroinformatics. 2014 Jun 22

21. Perani D, Della Rosa PA, Cerami c, Gallivanone F, Fallanca F, Vanoli EG, Panzacchi A,Nobili F, Pappatà S, Marcone A, Garibotto V, Castiglioni I, Magnani G, . Cappa SF, Gianolli G.Validation of an optimized SPM procedure for FDG-PET in dementia diagnosis in a clinical setting. NeuroImage Clinical, in press

22.Morris JC. The Clinical Dementia Rating (CDR): current version and scoring rules. Neurology $1993 ; 43: 2412-4$.

23.Chan D, Anderson V, Pijnenburg Y, Whitwell J, Barnes J, Scahill R, et al. The clinical profile of right temporal lobe atrophy. Brain 2009; 132:1287-98.

24. Josephs KA, Whitwell JL, Knopman DS, Boeve BF, Vemuri P, Senjem ML, et al. Two distinct subtypes of right temporal variant frontotemporal dementia. Neurology 2009; 73:1443-50.

25.Coombs BD, Szumowski J, Coshow W. Two-point Dixon technique for water-fat signal decomposition with B0 inhomogeneity correction. Magn Reson Med 1997; 38:884-9.

26. Hudson HM, Larkin RS. Accelerated image reconstruction using ordered subsets of projection data/ IEEE Trans Med Imaging; 1994: 13:601-9.

27. Wahlund LO, Julin P, Lindqvist J, Scheltens P. Visual assessment of medical temporal lobe atrophy in demented and healthy control subjects: correlation with volumetry. Psychiatry Res 1999; 90:193-9.

28.Möller C, Van der Flier WM, Versteeg A, Benedictus MR, Wattjes MP, Koedam EL, et al. Quantitative regional validation of the visual rating scale for posterior cortical atrophy. Eur 
Radiol 2013; 24:397-404.

29.Kipps CM, Davies RR, Mitchell J, Kril JJ, Halliday GM, Hodges JR. Clinical significance of lobar atrophy in frontotemporal dementia: application of an MRI visual rating scale. Dement Geriatr Cogn Disord 2007; 23:334-42.

30. Fischl B. FreeSurfer. Neuroimage 2012; 62:774-81.

31.Desikan RS, Ségonne F, Fischl B, Quinn BT, Dickerson BC, Blacker D, et al. An automated labeling system for subdividing the human cerebral cortex on MRI scans into gyral based regions of interest. Neuroimage 2006; 31:968-80.

32.Patenaude B, Smith SM, Kennedy DN, Jenkinson M. A Bayesian model of shape and appearance for subcortical brain segmentation. Neuroimage 2011; 56:907-22.

33. Morey RA, Petty CM, Xu Y, Pannu Hayes J, Wagner HR II, Lewis DV, et al. A comparison of automated segmentation and manual tracing for quantifying hippocampal and amygdala volumes. Neuroimage 2009; 45:855-66.

34.Sánchez-Benavides G, Gómez-Ansón B, Sainz A, Vives Y, Delfino M, Peña-Casanova J. Manual validation of FreeSurfer's automated hippocampal segmentation in normal aging, mild cognitive impairment, and Alzheimer Disease subjects. Psychiatry Res 2010; 181:21925.

35. Mulder ER, de Jong RA, Knol DL, van Schijndel RA, Cover KS, Visser PJ, et al. Hippocampal volume change measurement: Quantitative assessment of the reproducibility of expert manual outlining and the automated methods FreeSurfer and FIRST. Neuroimage 2014; 92:169-81.

36. Minoshima S, Frey KA, Koeppe RA, Foster NL, Kuhl DE. A diagnostic approach in Alzheimer's disease using three-dimensional stereotactic surface projections of fluorine-18FDG PET. J Nucl Med 1995; 36:1238-48.

37.Talairach $\mathrm{J}$ and Tournoux $\mathrm{P}$. Co-planar stereotaxic atlas of the human brain: 3-Dimensional proportional system: an approach to cerebral imaging. New York: Thieme Medical Publishers; 1988.

38.Perani D, Della Rosa PA, Cerami C, Gallivanone F, Fallanca F, Vanoli EG, Panzacchi A, Nobili F, Pappatà A, Marcone A, Garibotto V, Castiglioni I, Magnani G, Cappa SF, Gianolli F. Validation of an optimized SPM procedure for FDG-PET in dementia diagnosis in a clinical setting. NeuroImage Clinical 2014, in press

39.Signorini M, Paulesu E, Friston K, Perani D, Colleluori A, Lucignani G, Grassi F, Bettinardi V, Frackowiak RS, Fazio F.Rapid assessment of regional cerebral metabolic abnormalities in single subjects with quantitative and nonquantitative [18F]FDG PET: A clinical validation of 
statistical parametric mapping.Neuroimage. 1999;9:63-80.

40.Cohen J. A Coefficient of Agreement for Nominal Scales. Educ Psychol Meas 1960; 20:3746.

41.Warrens MJ. On Association Coefficients for $2 \times 2$ Tables and Properties That Do Not Depend on the Marginal Distributions. Psychometrika 2008; 73:777-89.

42.Fleiss JL. Measuring nominal scale agreement among many raters. Psychological Bulletin $1971 ; 76: 378-82$.

43.Dukart J, Mueller K, Villringer A, et al. Relationship between imaging biomarkers, age, progression and symptom severity in Alzheimer's disease. Neuroimage Clin 2013; 3:84-9

44.Jack CR Jr, Knopman DS, Jagust WJ, Petersen RC, Weiner MW, Aisen PS, Shaw LM, Vemuri P, Wiste HJ, Weigand SD, Lesnick TG, Pankratz VS, Donohue MC, Trojanowski JQ. Tracking pathophysiological processes in Alzheimer's disease: an updated hypothetical model of dynamic biomarkers. Lancet Neurol. 2013;12:207-16.

45. Mesulam MM. Primary progressive aphasia. Ann Neurol 2001; 49:425-32.

46.Lehmann M, Ghosh PM, Madison C, Laforce R, Corbetta-Rastelli C, Weiner MW, et al. Diverging patterns of amyloid deposition and hypometabolism in clinical variants of probable Alzheimer's disease. Brain 2013; 136:844-58.

47.Josephs KA, Duffy JR, Fossett TR, Strand EA, Claassen DO, Whitwell JL, et al. Fluorodeoxyglucose F18 positron emission tomography in progressive apraxia of speech and primary progressive aphasia variants. Arch Neurol 2010; 67:596-605.

48. Teune LK, Bartels AL, de Jong BM, Willemsen AT, Eshuis SA, de Vries JJ, van Oostrom JC, Leenders KL.Typical cerebral metabolic patterns in neurodegenerative brain diseases.Mov Disord. 2010;25:2395-404.

49. Samuraki M, Matsunari I, Chen W-P, Yajima K, Yanase D, Fujikawa A, et al. Partial volume effect-corrected FDG PET and grey matter volume loss in patients with mild Alzheimer's disease. Eur J Nucl Med Mol Imaging 2007; 34:1658-69.

50.Frisoni GB, Bocchetta M, Chételat G, Rabinovici GD, de Leon MJ, Kaye J, Reiman EM, Scheltens P, Barkhof F, Black SE, Brooks DJ, Carrillo MC, Fox NC, Herholz K, Nordberg A, Jack CR Jr, Jagust WJ, Johnson KA, Rowe CC, Sperling RA, Thies W, Wahlund LO, Weiner MW, Pasqualetti P, Decarli C; ISTAART's NeuroImaging Professional Interest Area. Imaging markers for Alzheimer disease: which vs how. Neurology. 2013; 81:487-500. 
Figure 1. Combined T1-MRI, PET uptake and SPM maps (a) and NEUROSTAT projections (b) from a 71-year old male with probable Alzheimer's disease (case no. 2). Moderate hippocampal atrophy is observed, alongside severe hypometabolism in retrosplenial, lateral parietal and dorsolateral prefrontal cortex. Medial temporal structures have normal metabolism on SPM maps, which, by comparison with uptake maps, emphasize the involvement of extra-temporal regions. NEUROSTAT projections reveal overall similar changes, but with limited anatomical specificity. Sections in (a) are shown in radiological convention (left side corresponds to right hemisphere), whereas NEUROSTAT projections (b) are shown in neurological convention (left side corresponds to left hemisphere).

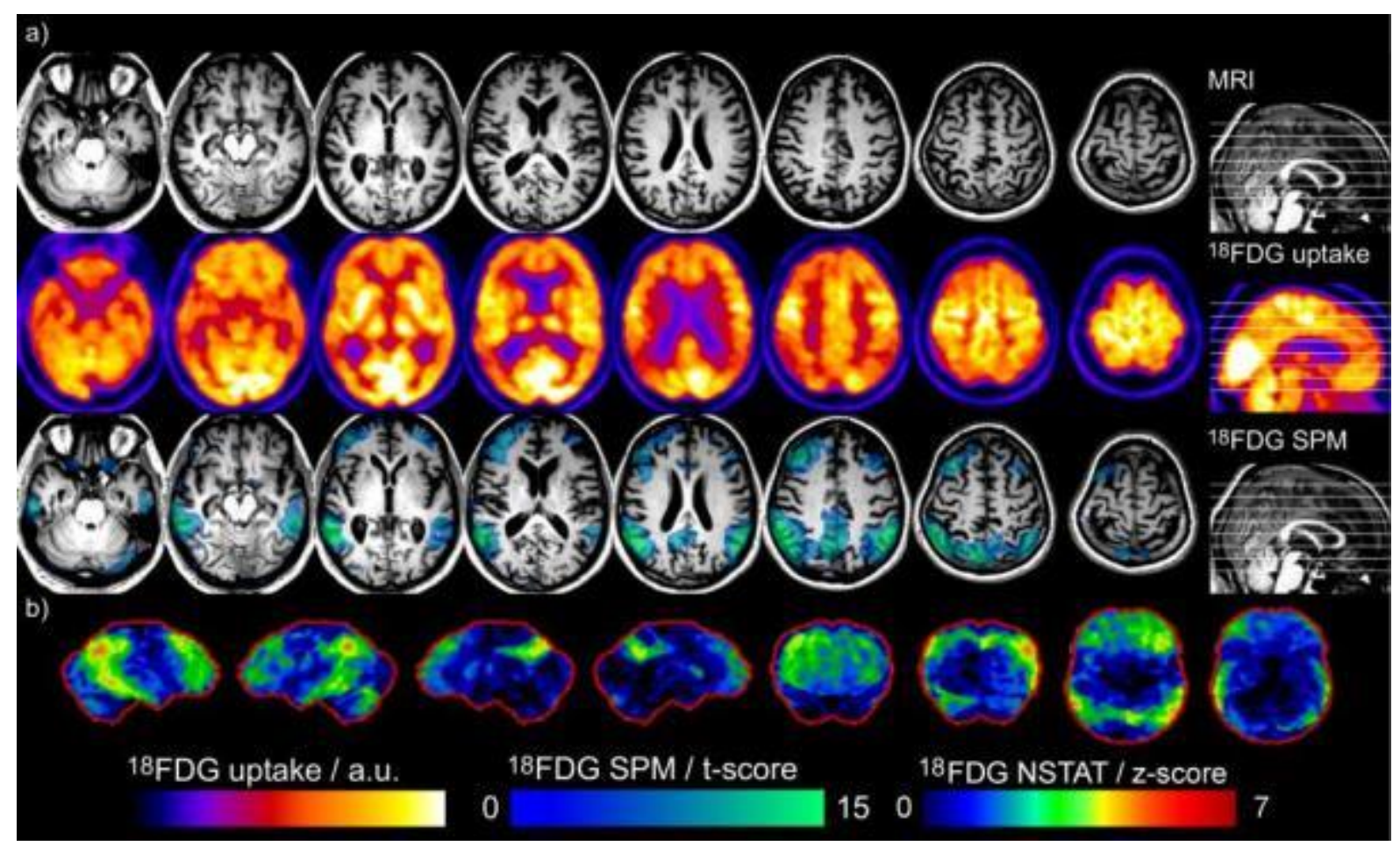


Figure 2.Combined T1-MRI, PET uptake and SPM maps (a) and NEUROSTAT projections (b) from a 61-year-old female with posterior cortical atrophy (case no. 8). Severe parietal atrophy and hypometabolism are concurrently observed. PET uptake maps, SPM maps and NEUROSTAT projections additionally show hypometabolism in medial parietal, posterior cingulate, left dorsolateral prefrontal and superior temporal cortex.Sections in (a) are shown in radiological convention (left side corresponds to right hemisphere), whereas NEUROSTAT projections (b) are shown in neurological convention (left side corresponds to left hemisphere).

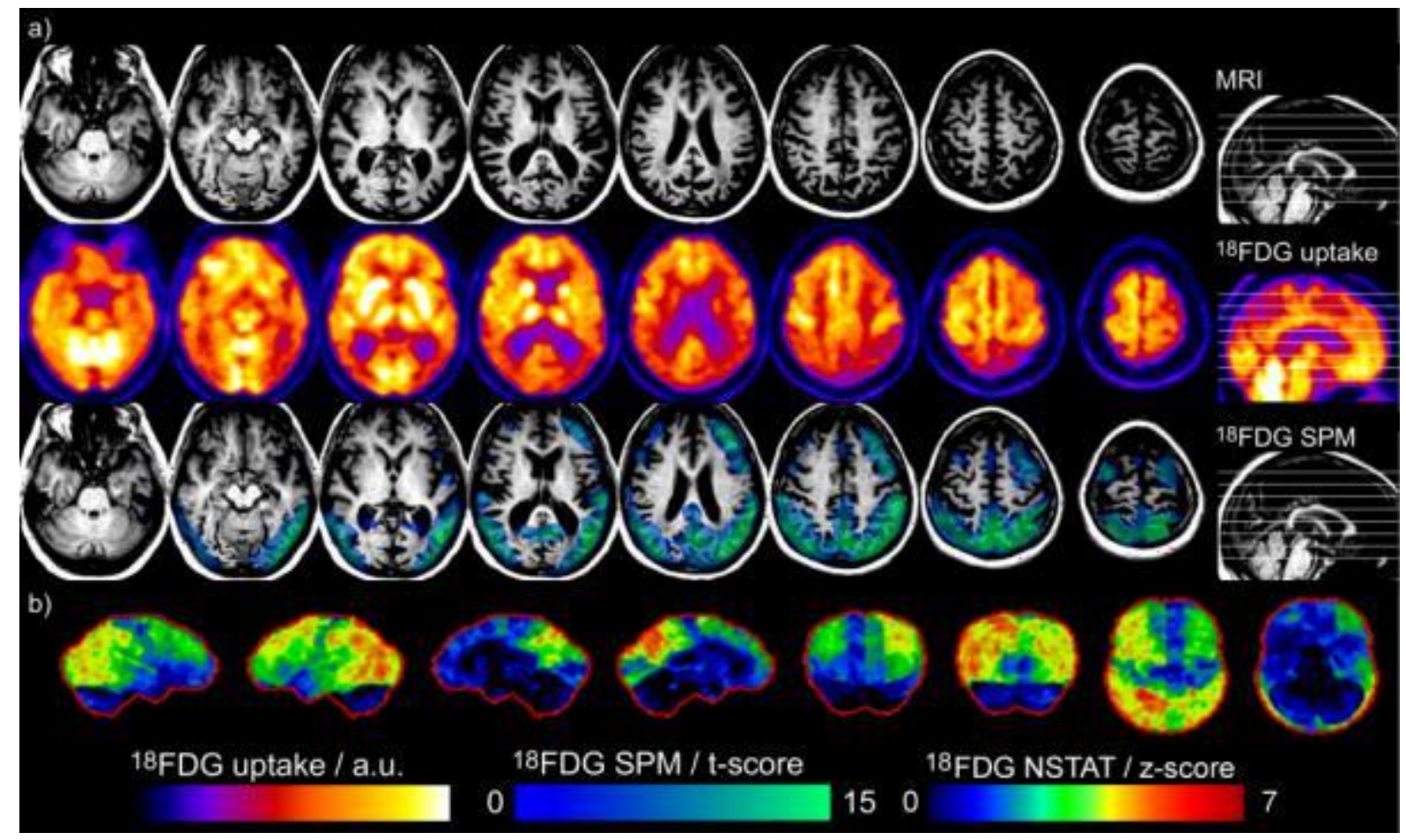


Figure 3. Combined T1-MRI, PET uptake and SPM maps (a) and NEUROSTAT projections (b) from a 67-year-old female with probable behavioural variant fronto-temporal dementia (case no. 10).

Medial frontal atrophy is observed with co-localised hypometabolism on PET uptake and SPM maps and NEUROSTAT projections. SPM maps and NEUROSTAT projections additionally demonstrate mild involvement of lateral parietal regions and caudate nuclei. Sections in (a) are shown in radiological convention (left side corresponds to right hemisphere), whereas NEUROSTAT projections (b) are shown in neurological convention (left side corresponds to left hemisphere).

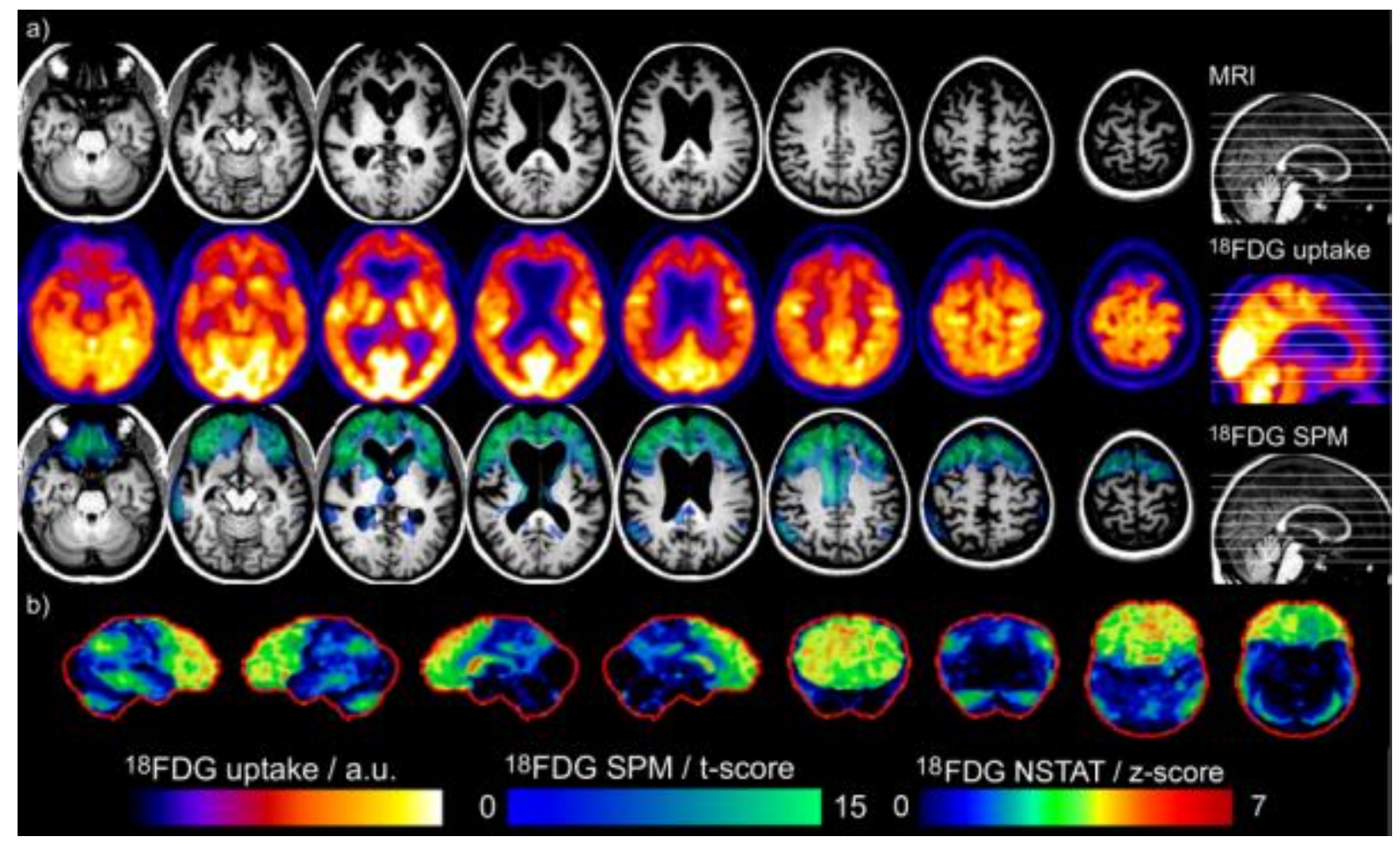


Figure 4.Combined T1-MRI, PET uptake and SPM maps (a) and NEUROSTAT projections (b) from a 63-year-old male with progressive non-fluent aphasia (case no. 16). Mild atrophy of the left Sylvian fissure is observed, while the SPM PET maps reveal hypometabolism in lateral temporo-parietal and frontal regions predominantly on the left. Uptake maps and NEUROSTAT projections show comparable effects.Sections in (a) are shown in radiological convention (left side corresponds to right hemisphere), whereas NEUROSTAT projections (b) are shown in neurological convention (left side corresponds to left hemisphere).

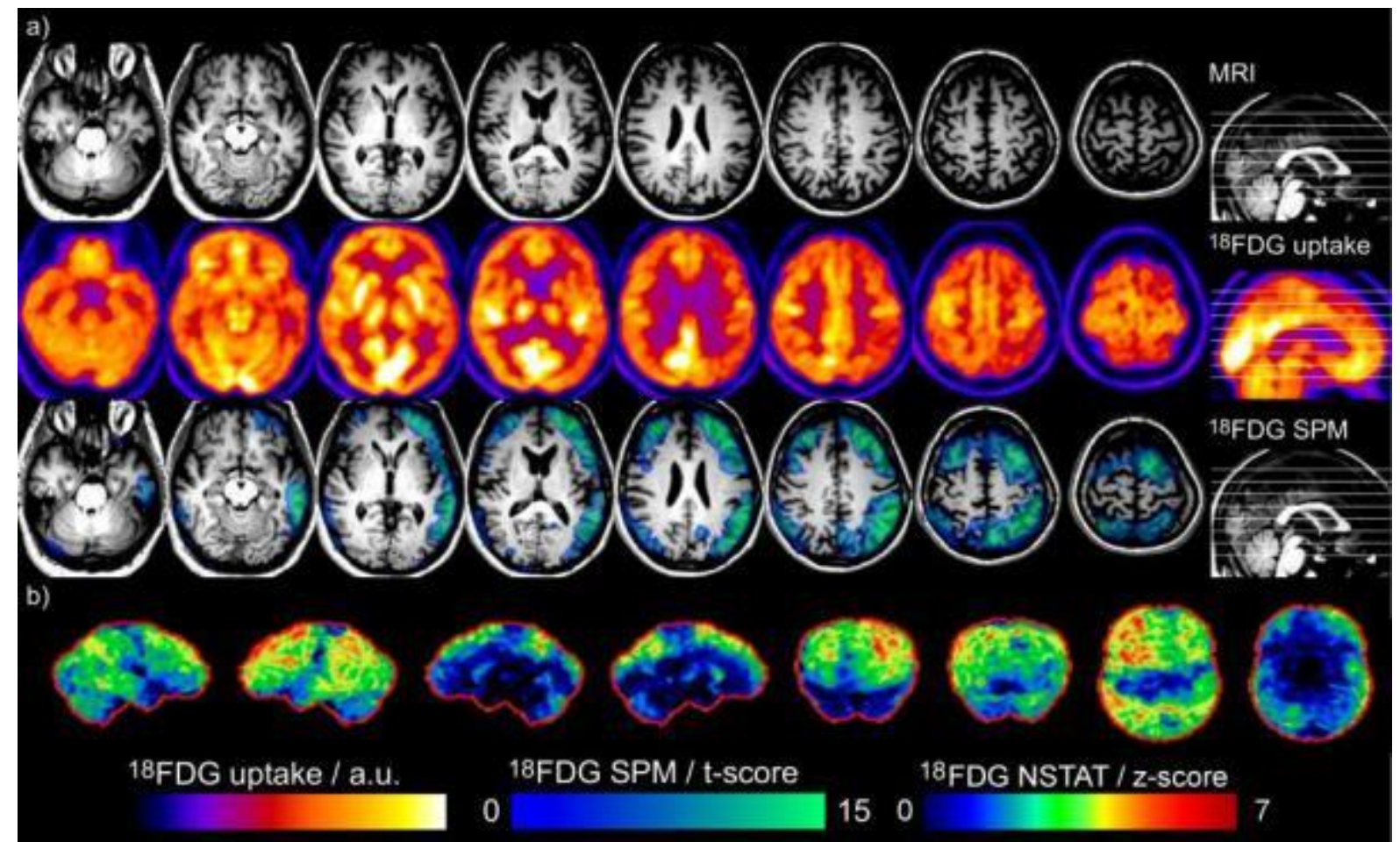


Figure 5. Combined T1-MRI, PET uptake and SPM maps (a) and NEUROSTAT projections (b) from a 72-year-old male with semantic variant of primary progressive aphasia (case no. 17). Bilateral antero-medial temporal lobe and dorsolateral frontal atrophy are observed, predominantly on the left. The corresponding SPM PET maps highlight predominantly left-sided hypometabolism in medial and lateral temporal, inferior parietal and medial frontal regions with additional involvement of the left ventral tegmentum and caudate nuclei. This diffuse pattern of hypometabolism is not observed on uncorrected uptake maps and NEUROSTAT projections, however the changes in the temporal regions are observed. Sections in (a) are shown in radiological convention (left side corresponds to right hemisphere), whereas NEUROSTAT projections (b) are shown in neurological convention (left side corresponds to left hemisphere).

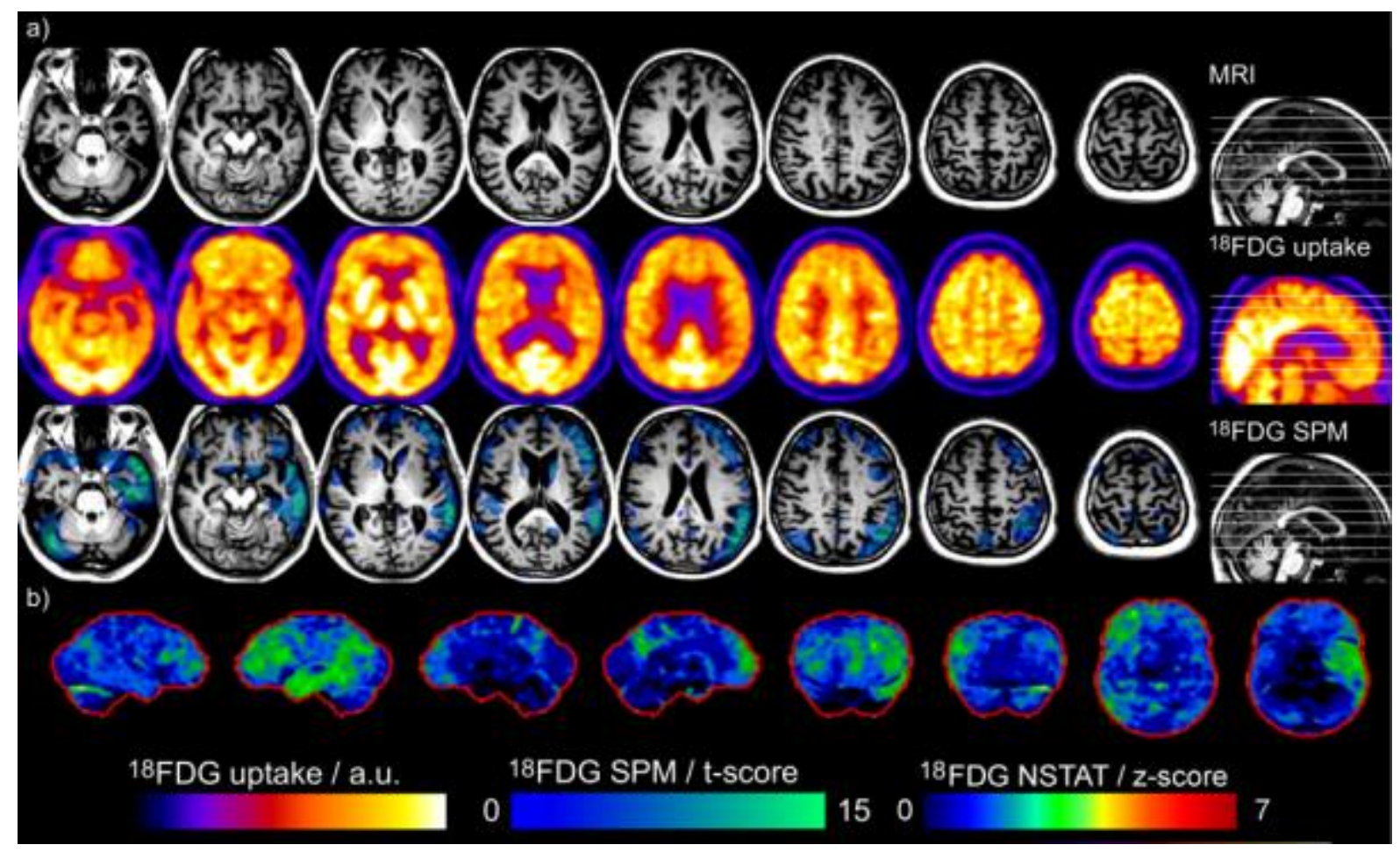


Figure 6. Combined T1-MRI, PET uptake and SPM maps (a) and NEUROSTAT projections (b) from a 57-year-old male with right temporal variant fronto-temporal dementia (case no. 23). Focal right medial temporal atrophy and more weakly dorsolateral prefrontal atrophy are observed. SPM PET maps highlight severe inferior-lateral temporal and parietal hypometabolism, with moderate changes also in right precuneus and cingulate cortex. The corresponding uptake maps and NEUROSTAT projections highlight similar changes but are less definitive in terms of localization ofcingulate and frontal changes. Sections in (a) are shown in radiological convention (left side corresponds to right hemisphere), whereas NEUROSTAT projections (b) are shown in neurological convention (left side corresponds to left hemisphere).

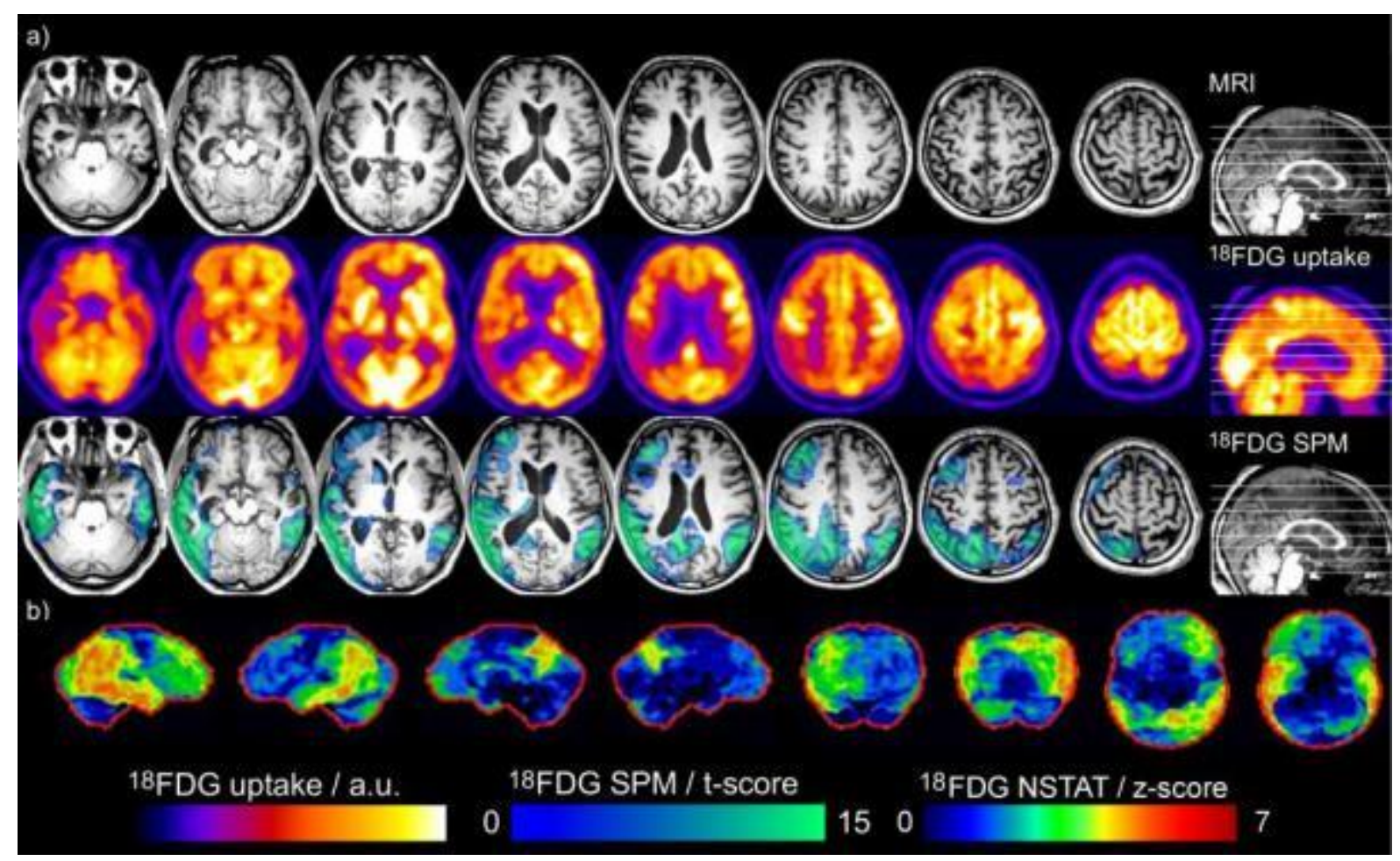


Supplementary Figure 1. Combined T1-MRI, PET uptake and SPM maps (left) and atrophy and hypometabolism ratings (right) in Alzheimer' s disease. Hippocampal atrophy is associated with posterior cingulate and lateral temporo-parietal hypometabolism. The dorsolateral frontal and medial parietal hypometabolism observed in cases no. 1-3 contrast with the medial temporal hypometabolism observed in case no. 4. Occipital lobe hypometabolism appearsdecoupled from the severe precuneus hypometabolism found in cases no. 1,2 and 3.For images and plots, left side corresponds to left hemisphere.
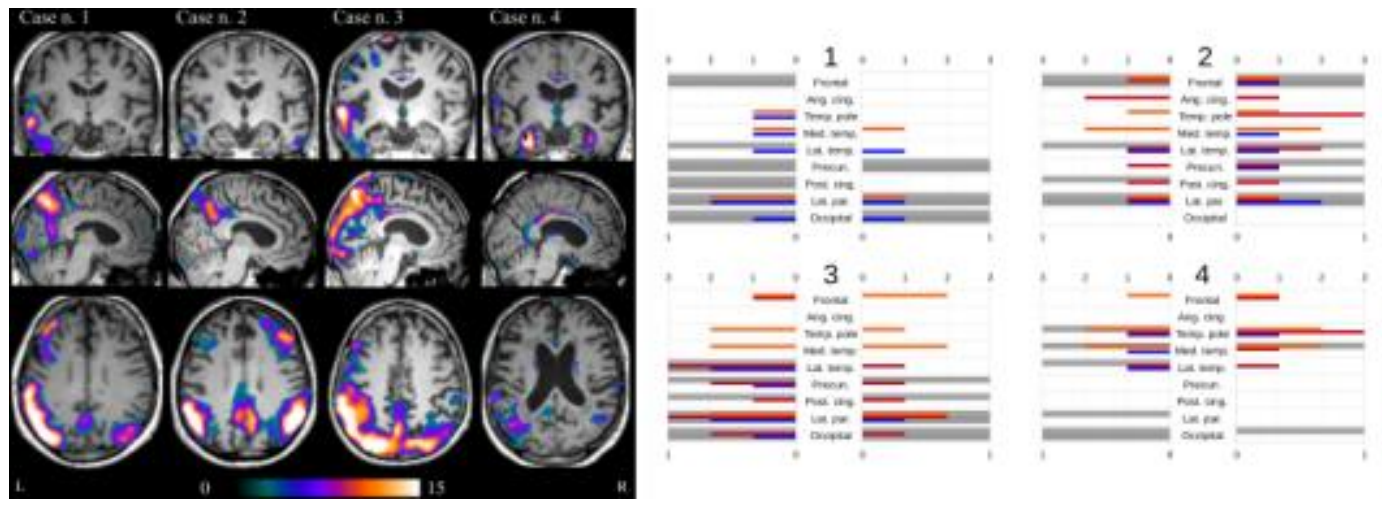
Supplementary Figure 2. Combined T1-MRI, PET uptake and SPM maps (left) and atrophy and hypometabolism ratings (right) in posterior cortical atrophy. Co-localised parietal atrophy and hypometabolism are observed, with additional hypometabolism in lateral temporal and dorsolateral frontal regions. Occipital hypometabolism is noted in cases with primary visual failure (cases no. 5, 6 and 8); by comparison, the case with biparietal syndrome (case no. 7) demonstrates particularly marked hypometabolism over both parietal lobes, extending to the left post-central gyrus and supplementary motor area.For images and plots, left side corresponds to left hemisphere.
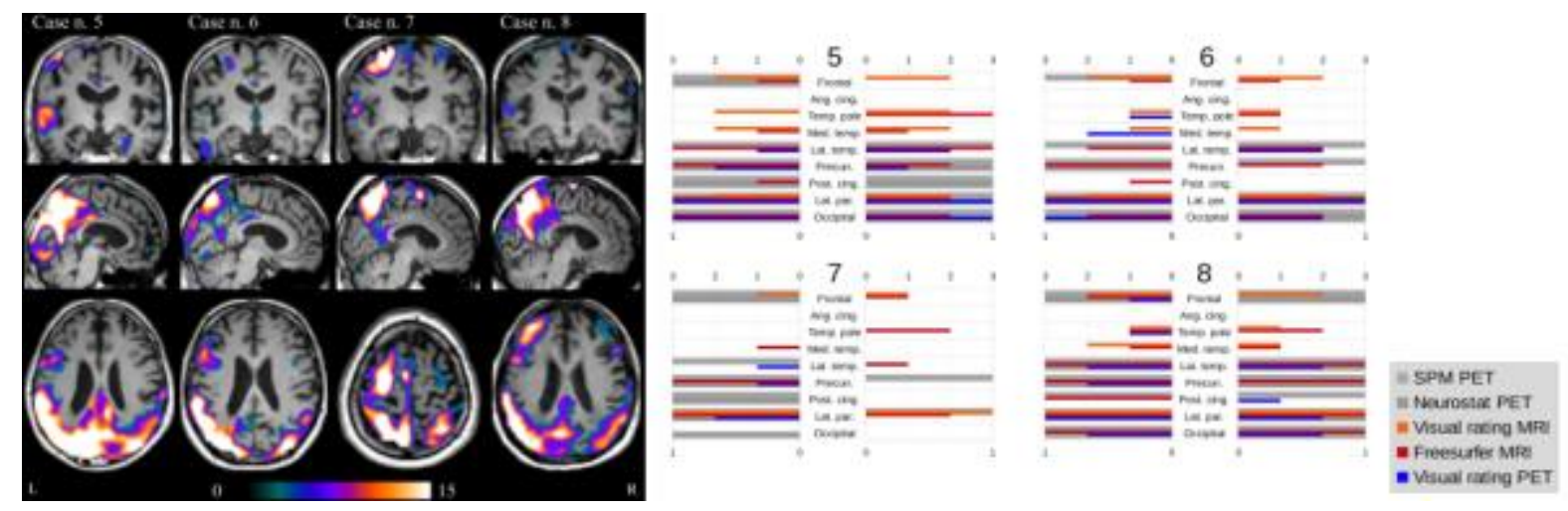
Supplementary Figure 3. Combined T1-MRI, PET uptake and SPM maps (left) and atrophy and hypometabolism ratings (right) in behavioural variant fronto-temporal dementia. Frontal atrophy is accompanied by medial frontal and parietal hypometabolism, however the topography of frontal lobe hypometabolism is distinct in each case. The anterior cingulate is involved in cases no.9, 10 and 12, whereas this is less evident in case no. 11, for which severe lateral fronto-parietal hypometabolism is observed, with marked involvement of the precuneus and posterior cingulate gyrus. For images and plots, left side corresponds to left hemisphere.
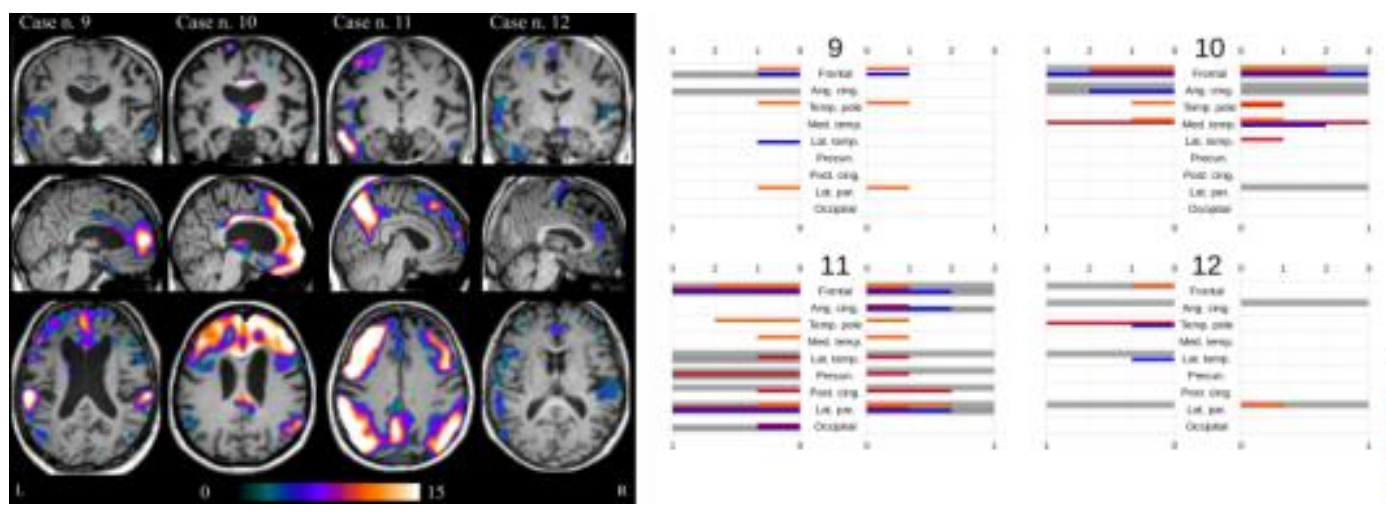
Supplementary Figure 4. Combined T1-MRI, PET uptake and SPM maps (left) and atrophy and hypometabolism ratings (right) in progressive non-fluent aphasia. Left perisylvian atrophy of variable severity is accompanied by lateral frontal and lateral temporo-parietal hypometabolism, which was particularly marked in cases 14-16. For images and plots, left side corresponds to left hemisphere.
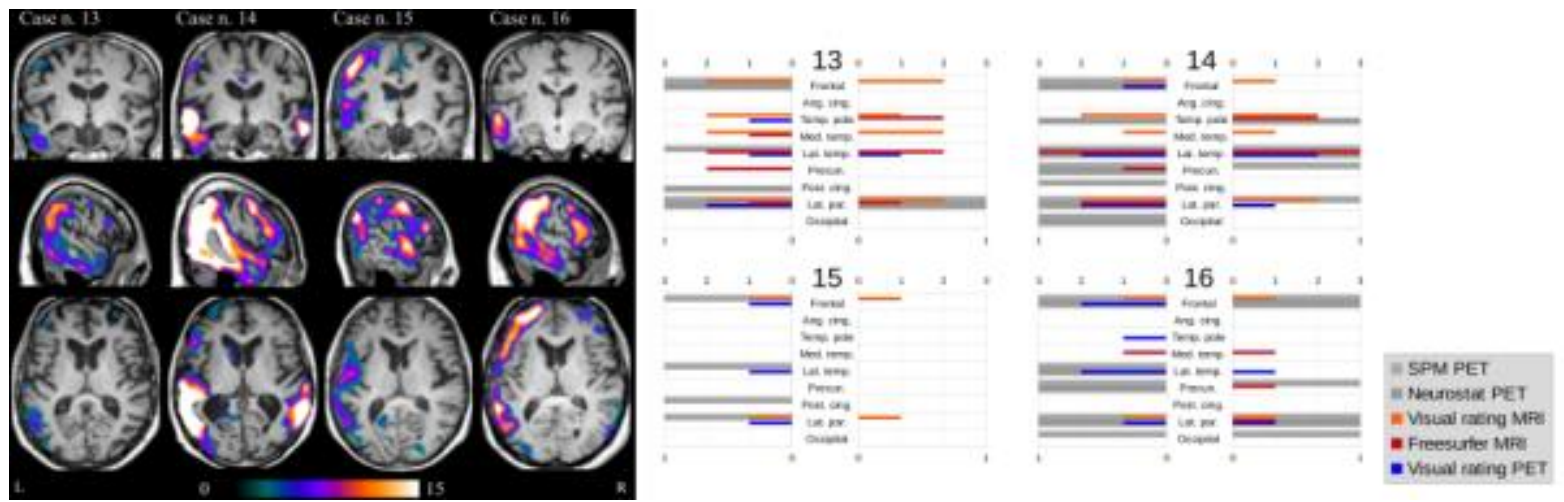
Supplementary Figure 5. Combined T1-MRI, PET uptake and SPM maps (left) and atrophy and hypometabolism ratings (right) in semantic variant of primary progressive aphasia. Left temporal atrophy and hypometabolism are consistently co-localized, but individual differences are observed especially with regards to anterior cingulate and medial frontal hypometabolism. Parietal atrophy and hypometabolism are predominantly observed in the left hemisphere.For images and plots, left side corresponds to left hemisphere.
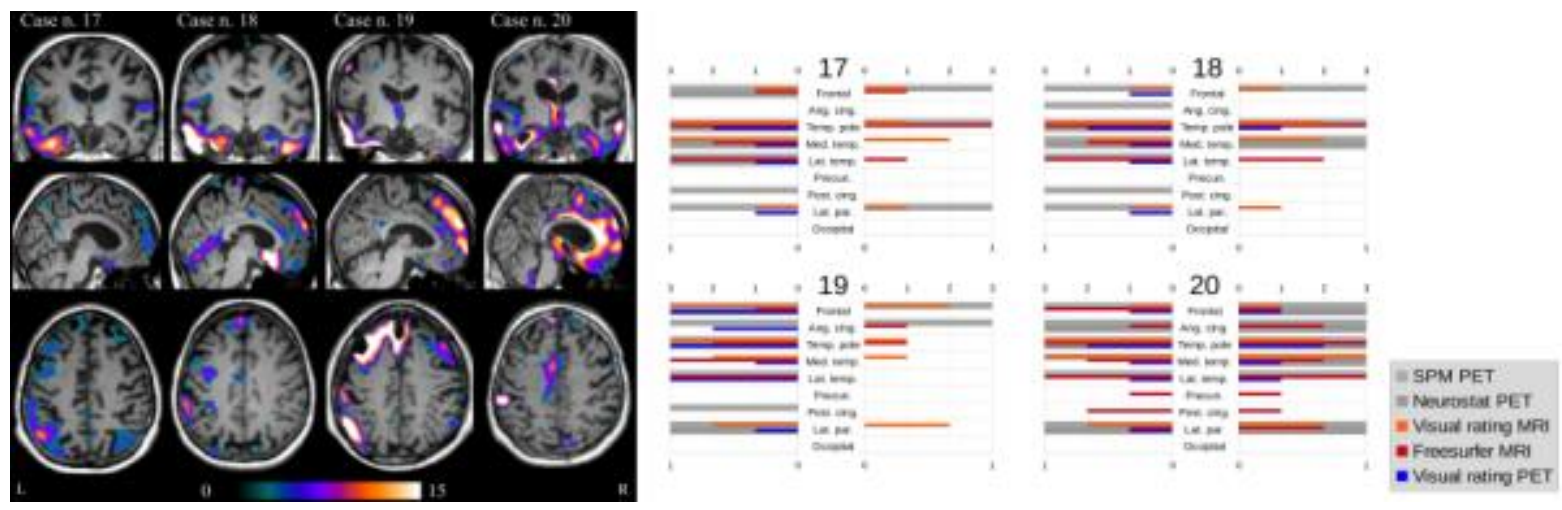
Supplementary Figure 6. Combined T1-MRI, PET uptake and SPM maps (left) and atrophy and hypometabolism ratings (right) in right temporal variant frontotemporal dementia. There is consistent co-localisation of atrophy and hypometabolism in the antero-medial temporal lobes and limbic cortex, with individual differences observed in terms of frontal lobe hypometabolism. The medial frontal and anterior cingulate hypometabolism observed in cases no. 21, 22 and 24 is contrast with the involvement of the dorsolateral frontal lobe, precuneus and posterior cingulate cortex in case no. 23 . For images and plots, left side corresponds to left hemisphere.
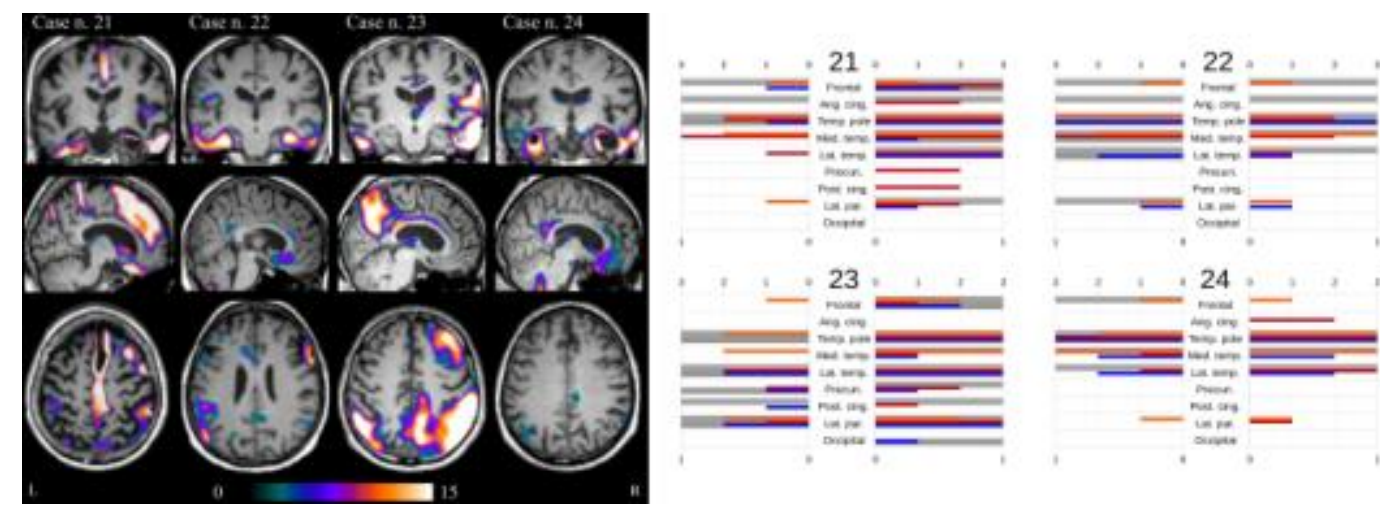


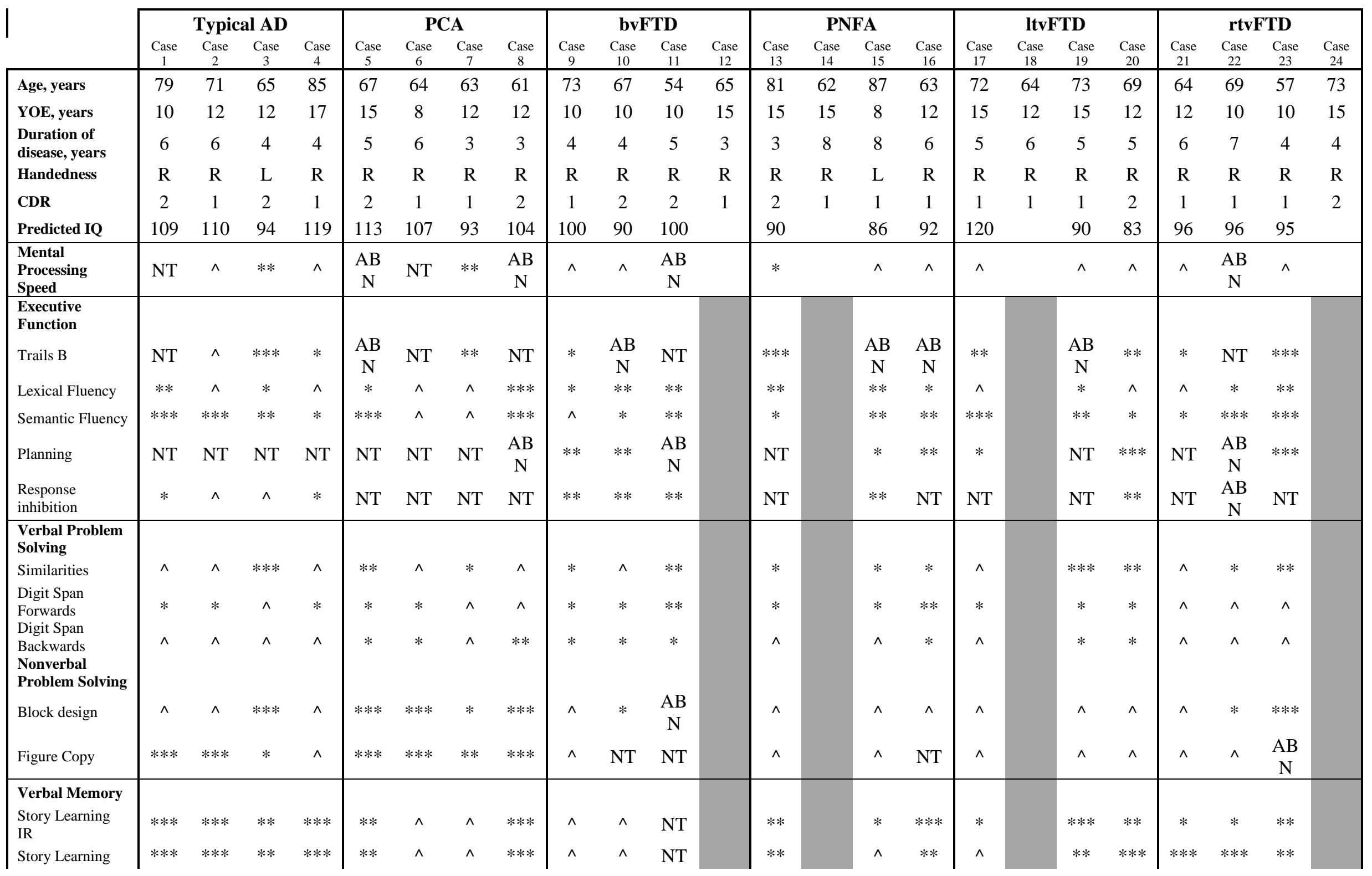




\begin{tabular}{|c|c|c|c|c|c|c|c|c|c|c|c|c|c|c|c|c|c|c|c|c|}
\hline \multicolumn{21}{|l|}{ DR } \\
\hline List Learning IR & NT & $* *$ & $* * *$ & $* * *$ & $* *$ & $\wedge$ & $\wedge$ & NT & NT & $*$ & $* * *$ & $* *$ & * & $* *$ & $\wedge$ & $* *$ & $* *$ & NT & $* *$ & $* * *$ \\
\hline $\begin{array}{l}\text { List Learning } \\
\text { LDR }\end{array}$ & NT & $* * *$ & $* *$ & $* *$ & $* *$ & $\wedge$ & $\wedge$ & NT & NT & $\wedge$ & $* *$ & $* *$ & $\wedge$ & $* *$ & $*$ & ** & $* *$ & NT & $* * *$ & $* *$ \\
\hline $\begin{array}{l}\text { List Learning } \\
\text { Recognition }\end{array}$ & NT & $* *$ & $* *$ & $* *$ & $* *$ & $\wedge$ & $*$ & NT & NT & $\wedge$ & $*$ & $* *$ & $\wedge$ & $*$ & $*$ & $*$ & $*$ & NT & $* * *$ & $* *$ \\
\hline \multicolumn{21}{|l|}{$\begin{array}{l}\text { Nonverbal } \\
\text { Memory }\end{array}$} \\
\hline $\begin{array}{l}\text { Figure immediate } \\
\text { recall }\end{array}$ & $*$ & $*$ & $* * *$ & $* *$ & $* * *$ & $* *$ & $\wedge$ & NT & $\wedge$ & NT & NT & $\wedge$ & $\wedge$ & NT & $* *$ & $\wedge$ & $*$ & $*$ & $\wedge$ & NT \\
\hline $\begin{array}{l}\text { Figure delayed } \\
\text { recall }\end{array}$ & * & $*$ & $* * *$ & $* * *$ & $* * *$ & $* *$ & $\wedge$ & NT & $\wedge$ & NT & NT & $\wedge$ & $\wedge$ & NT & $*$ & $\wedge$ & $\wedge$ & $*$ & $* *$ & NT \\
\hline $\begin{array}{l}\text { Delayed Facial } \\
\text { Recall }\end{array}$ & $* *$ & $* *$ & NT & $*$ & NT & $\wedge$ & NT & $*$ & $\wedge$ & NT & NT & NT & NT & NT & $*$ & $\wedge$ & $\wedge$ & $* * *$ & $* * *$ & $* * *$ \\
\hline $\begin{array}{l}\text { Confrontational } \\
\text { Naming } \\
\end{array}$ & $*$ & $*$ & $* *$ & $*$ & $* * *$ & $* *$ & $\wedge$ & $* *$ & $* *$ & $\wedge$ & $\wedge$ & $* *$ & $* *$ & $* *$ & $* * *$ & $* * *$ & $* * *$ & $* * *$ & $* * *$ & $* * *$ \\
\hline \multicolumn{21}{|l|}{ Visuospatial } \\
\hline VOSP screening & NT & $\wedge$ & $\wedge$ & NT & $\wedge$ & $\wedge$ & $\wedge$ & $\wedge$ & NT & $\wedge$ & $\wedge$ & $\wedge$ & NT & NT & $\wedge$ & NT & $\wedge$ & $\wedge$ & NT & $\wedge$ \\
\hline $\begin{array}{l}\text { VOSP dot } \\
\text { counting }\end{array}$ & NT & NT & $* *$ & NT & $* *$ & NT & $\wedge$ & NT & NT & $\wedge$ & $\wedge$ & $\wedge$ & NT & NT & $\wedge$ & NT & $\wedge$ & $\wedge$ & NT & $\wedge$ \\
\hline $\begin{array}{l}\text { VOSP number } \\
\text { location }\end{array}$ & NT & $\wedge$ & $\wedge$ & NT & NT & $* * *$ & $\wedge$ & NT & NT & $\wedge$ & NT & $\wedge$ & NT & NT & NT & NT & $\wedge$ & $\wedge$ & NT & $\wedge$ \\
\hline $\begin{array}{l}\text { VOSP object } \\
\text { decision }\end{array}$ & NT & NT & NT & NT & $* * *$ & $* * *$ & $\wedge$ & $* * *$ & NT & NT & $* *$ & NT & NT & NT & NT & NT & NT & NT & NT & NT \\
\hline $\begin{array}{l}\text { VOSP cube } \\
\text { analysis }\end{array}$ & NT & $\wedge$ & NT & NT & NT & $* * *$ & $* *$ & $* * *$ & NT & NT & NT & NT & NT & NT & $\wedge$ & NT & NT & NT & NT & NT \\
\hline
\end{tabular}

Supplementary Table 1. Psychometric data. Individual test scores (standard scores and/or percentile ranks) were expressed as t-scores and ordered using a four-point system: t-score > 42: unimpaired, 37-42: *, impairment in the 10-25th centile, 29-26: **, impairment in the 2nd-9th centile, <26: *** (impairment below the 2nd centile). ABN: abandoned. NT: Not tested. 